\title{
Procreative beneficence and in vitro gametogenesis
}

\author{
Hannah Bourne \\ Monash University Medical School \\ Thomas Douglas \\ Oxford Uehiro Centre for Practical Ethics, University of Oxford \\ Julian Savulescu \\ Oxford Uehiro Centre for Practical Ethics, University of Oxford \\ julian.savulescu@philosophy.ox.ac.uk
}

The Principle of Procreative Beneficence (PB) holds that when a couple plans to have a child, they have significant moral reason to select, of the possible children they could have, the child who is most likely to experience the greatest wellbeing - that is, the most advantaged child, the child with the best chance at the best life. ${ }^{1} \mathrm{~PB}$ captures the common sense intuitions of many about reproductive decisions. For example, many couples defer conceiving until they are in a better financial or social situation in an attempt to give their child a better life. Or a woman who is currently suffering from rubella may defer conception until she has recovered to prevent giving birth to a severely impaired child. In both of these cases, parents are choosing to have one child, who can expect a better life, over another, who could expect a worse life. Most people believe that such choices are morally permissible, if not morally required. $\mathrm{PB}$ extrapolates from our intuitions about such cases to cases involving selection using technological means, such as pre-implantation genetic diagnosis.

$\mathrm{PB}$ does not posit an absolute moral obligation - it does not dictate what people must do. Instead it holds that there is a significant moral reason to select the best child, but one that must be weighed against other reasons.

Julian Savulescu, 'Procreative Beneficence: Why We Should Select the Best Children', Bioethics 15 (5-6), 2001, 413-26; Julian Savulescu and Guy Kahane, 'The Moral Obligation to Create Children with the Best Chance of the Best Life', Bioethics 23 (5), 2009, 274-90. 
Many objections have been raised to $\mathrm{PB}$. For example, it has been held that adopting the principle would exacerbate inequalities, since the wealthy would have better access to selection technologies than the poor; ${ }^{2}$ would reinforce objectionable societal preferences (such as preferences for children of a certain sex or sexual orientation); ${ }^{3}$ would impose unacceptable burdens on women ${ }^{4}$ and on parents in general; ${ }^{5}$ would result in a generation of disillusioned children, unable to achieve what is expected of them; ${ }^{6}$ and would generate an 'arms-race' as parents iteratively attempt to prevent their own children from being placed at a competitive disadvantage. ${ }^{7}$ The principle has also been criticised for failing to give parents all-things-considered guidance on what to $\mathrm{do}^{8}$ failing to bridge the gap between what is best, and what parents think is best; ${ }^{9}$ ignoring the complexity and contextdependence of the good life; ${ }^{10}$ and being excessively individualistic. ${ }^{11}$ Savulescu and collaborators have responded to some of these objections elsewhere. ${ }^{12}$ In this paper we wish address a different and more practical objection: the objection that parents will be heavily restricted in the number of traits that they can select, since they will have to choose among a very limited number of embryos. Recent advances in stem cell research may provide a solution to this problem.

\section{In vitro gametogenesis}

Recent research suggests that it may become possible to derive gametes (eggs and sperm) from human stem cells in vitro, a process which we will term in

\footnotetext{
Savulescu, 'Procreative Beneficence'.

3 Ibid.; Savulescu and Kahane, 'The Moral Obligation to Create Children with the Best Chance of the Best Life'; Inmaculada de Melo-Martin, 'On Our Obligation to Select the Best Children: A Reply to Savulescu', Bioethics 18 (1), 2004, 72-83.

4 De Melo-Martin, 'A Reply to Savulescu'.

6 Kean Birch, 'Beneficence, Determinism and Justice: an engagement with the argument for the genetic selection of intelligence', Bioethics 19 (1), 2005,12-28.

7 De Melo-Martin, 'A Reply to Savulescu'; Birch, 'Beneficence, Determinism and Justice'.

8 De Melo-Martin, 'A Reply to Savulescu'.

10 Ibid.; Birch, 'Beneficence, Determinism and Justice'.

11 Birch, 'Beneficence, Determinism and Justice'.

12 Savulescu and Kahane, 'The Moral Obligation to Create Children with the Best Chance of the Best Life'.
}

5 Ibid.

9 Ibid. 
vitro gametogenesis (IVG). IVG would allow the creation of stems cells from a patient's somatic (body) cells, and these stems cells could then be used to generate a plentiful supply of eggs or sperm in the laboratory.

Major advances in this technology have been made in the mouse. Both spermlike ${ }^{13}$ and egg-like ${ }^{14}$ cells have been derived from murine (mouse) embryonic stem cells (mESCs) in vitro. One laboratory also reported the production of live offspring following fertilization of natural mouse eggs with sperm-like cells derived from mESCs. ${ }^{15}$ Another laboratory was able to produce both spermlike and egg-like cells from mESCs in the same Petri dish. ${ }^{16}$ Progress towards IVG in humans has been slower, ${ }^{17}$ but researchers have been able to derive cells expressing markers specific to mature germ cells from human ES cells. ${ }^{18}$ Testing the reproductive functionality of these cells is, however, challenging due to ethical constraints placed on human research.

IVG, based on this research, would involve a number of steps. One possibility is that the genetic material from a somatic (body) cell from a patient would be transferred into an enucleated oocyte (an egg that has had its nucleus removed) via somatic cell nuclear transfer (SCNT), creating an

13 Niels Geijsen et al., 'Derivation of embryonic germ cells and male gametes from embryonic stem cells', Nature 427 (6970), 2004, 148-154; Yayoi Toyooka et al., 'Embryonic stem cells can form germ cells in vitro', Proceedings of the National Academy of Sciences 100 (20), 2003, 11457-62; Karim Nayernia et al., 'In Vitro-Differentiated Embryonic Stem Cells Give Rise to Male Gametes that Can Generate Offspring Mice', Developmental Cell 11(1), 2006, 125-32.

14 Karin Hübner et al., 'Derivation of Oocytes from Mouse Embryonic Stem Cells', Science 300 (5623), 2003, 1251-6; Orly Lacham-Kaplan, Hun Chy and Alan Trounson, 'Testicular Cell Conditioned Medium Supports Differentiation of Embryonic Stem Cells into Ovarian Structures Containing Oocytes', Stem Cells, 24 (2), 2006, 266-73.

15 Nayernia et al., 'In Vitro-Differentiated Embryonic Stem Cells'.

16 Alexandre Kerkis et al., 'In vitro differentiation of male mouse embryonic stem cells into both presumptive sperm cells and oocytes', Cloning and stem cells 9 (4), 2007, 535-48.

17 Please add ref to: Pelosi E, Forabosco A, Schlessinger D. 'Germ cell formation from embryonic stem cells and the use of somatic cell nuclei in oocytes', Annals of the New York Academy of Sciences 2011; 1221:18-26.

18 H F Chen et al., 'Derivation, characterization and differentiation of human embryonic stem cells: comparing serum-containing versus serum-free media and evidence of germ cell differentiation', Human Reproduction 22 (2), 2007, 567-77; Amanda Clark et al., 'Spontaneous differentiation of germ cells from human embryonic stem cells in vitro', Human Molecular Genetics 13 (7), 2004, 727-39. 
embryo which would be a clone of the patient. This cloned embryo would then be allowed to develop to the blastocyst stage. Developing germ cells (immature gametes) would then be harvested from the inner cell mass of the embryo and placed in culture to mature. Once the mature gamete had formed, the remainder of the procedure would follow current IVF techniques (fertilization followed by implantation).

Alternatively, new research suggests a method that would avoid the need for SCNT. It has been shown that adult mouse fibroblasts can be dedifferentiated to form immature cells that have characteristics of ES cells. These are known as induced pluripotent stem (iPS) cells. ${ }^{19}$ More recently, and more importantly, it has been shown that adult human fibroblasts can also form iPS cells. ${ }^{20}$ Drusenheimer et al. were also able to transdifferentiate human bone marrow cells into male sperm-like cells, ${ }^{21}$ while Dyce et al. utilized skin stem cells obtained from pig fetuses to derive egg-like cells. ${ }^{22}$ More recently, Hayashi et al. succeeded in producing fertile mice offspring using oocytes derived from mouse iPS cells. ${ }^{23}$ The ability to create iPS cells would bypass the need for SCNT. Stems cells could be derived directly from somatic cells, and could then be used to generate gametes.

IVG has four important foreseeable applications: to provide an in vitro model for the study of human gametogenesis (the formation of gametes) and

19 Kazutoshi Takahashi and Shinya Yamanaka, 'Induction of Pluripotent Stem Cells from Mouse Embryonic and Adult Fibroblast Cultures by Defined Factors', Cell 126 (4), 2006, 663-76; Masato Nakagawa et al. 'Generation of induced pluripotent stem cells without Myc from mouse and human fibroblasts', Nature Biotechnology 26 (1), 2008,101-6.

20 Kazutoshi Takahashi et al., 'Induction of Pluripotent Stem Cells from Adult Human Fibroblasts by Defined Factors', Cell 131 (5), 2006, 861-72; Park I, Zhao R, West Jea, 'Reprogramming of human somatic cells to pluripotency with defined factors', Nature 451 (7175), 2008, 141-6.

21 Nadja Drusenheimer et al., 'Putative human male germ cells from bone marrow stem cells', Society Of Reproduction of Fertility Supplement 63, 2007, 69-76.

22 Paul W Dyce, Lihua Wen and Julang $\mathrm{Li}$, 'In vitro germline potential of stem cells derived from fetal porcine skin', Nature Cell Biology 8 (4), 2006, 384-90.

23 Hayashi, Katsuhiko; Ogushi, Sugako, Kurimoto, Kazuki; Shimamoto, So; Ohta, Hiroshi; Saitou, Mitinori. 'Offspring from Oocytes Derived from in Vitro Primordial Germ CellLike Cells in Mice', Science , 4 October 2012. See: http://www.sciencemag.org/content/ early/2012/10/03/science.1226889. 
diseases of the germline; to create a supply of gametes for use in research or fertility treatment; and to enable genetic manipulation of the human germline. ${ }^{24}$

The main role of IVG in fertility treatment is likely to be the creation of a supply of eggs. While the natural supply of sperm is usually plentiful and easily obtainable, achieving a supply of eggs is much more problematic. Currently, if a woman wishes to employ assisted reproductive technologies, eggs must be harvested from her ovaries. This procedure is not without risk and discomfort. Furthermore, a limited number of eggs can be obtained via this method. IVG could remove the need to harvest eggs, since they could instead be derived and produced in abundance from somatic cells. In cases of infertility due to nonproduction of sperm, a similar approach could be used to produce sperm in large quantities.

The ability to create large numbers of eggs or sperm through IVG greatly increases our capacity to select the best child possible. Selection could occur in two ways: (1) the most genetically desirable of this massive number of gametes could be selected and then used to create an embryo, or alternatively, (2) large numbers of embryos could be produced from these gametes and then the best embryo selected. Whatever the method, the advent of IVG could allow us to select for a much larger number of traits than is currently conceivable. IVG may also offer new possibilities for genetic enhancement. Cells could be modified prior to gamete formation, most probably once an ES cell culture has been established. ${ }^{25}$ However, in this paper we focus on possibilities for selection, rather than enhancement.

24 George Q Daley, 'Gametes from Embryonic Stem Cells: A Cup Half Empty or Half Full?', Science 316 (5823), 2007, 409-10; Abby Lippman et al., 'The Ethics of Deriving Gametes from ES Cells', Science 307 (5709), 2005 515-7; Zubin Master, 'Embryonic stem-cell gametes: the new frontier in human reproduction', Human Reproduction 21 (4), 2006, 857-63; Anne McLaren, 'Free-Range Eggs?', Science 316 (5823), 2007, 339; A J Newson and A C Smajdor, 'Artificial Gametes: New Paths to Parenthood?', Journal of medical ethics 31(3), 2005, 184-6; M Azim Surani, 'Stem cells: How to make eggs and sperm', Nature 427 (6970), 2004, 106-7; Giuseppe Testa and John Harris, 'Ethics and Synthetic Gametes', Bioethics 19 (2), 2005, 146-66; Giuseppe Testa and John Harris, 'Ethical Aspects of ES Cell-Derived Gametes', Science 305 (5691), 2004, 1719; Peter Whittaker, 'Stem cells to gametes: how far should we go?', Human Fertility 10 (1), 2007, 1-5.

Whittaker, 'Stem cells to gametes'. 


\section{A simple model}

What effect would the ability to produce large numbers of gametes or embryos have on a couple's ability to select the most advantaged child? We answer this question using a simple mathematical model.

Suppose that a couple would like to select for 20 single gene traits which are carried on 20 different and unlinked autosomal loci. Suppose further that at ten of these loci, alleles contribute recessively to the desired trait. That is, of the four possible combinations of alleles - which we might label $R R, R r, r R$, and $r r$ - only $r r$ confers the disposition. Suppose that at the other ten loci, alleles contribute dominantly to the desired disposition, so that $D D, D d$, or $d D$ individuals will possess the disposition - only $d d$ individuals will lack it.

Not every couple in the population will be capable of having a child with the desired genotype at each locus. For example, suppose that a couple are $R R$ and $R r$ respectively at one of the recessive loci. It will be impossible for this couple to have a child with the desired $r r$ genotype at this locus. However, we can make some statistical observations about how likely it is that a randomly selected couple will be able to have a child with the desired genotype at a given locus. In general, if the four different allele combinations at each locus are equally prevalent in the population (male and female), then a typical couple will be capable of having a child with the desired genotype at 6 of the 10 recessive loci, and 9 of the 10 dominant loci [see Appendix 1]. Thus, a typical couple would be capable of having a child with 15 of the 20 desired traits.

Of course, the chance that a randomly selected couple would naturally have a child with all 15 desired traits is extremely small. The chance that the couple would have a child with the desired genotype at a given recessive locus at which the desired genotype is possible would be significant at 0.444444 . The probability that they would have a child with the desired genotype at a given dominant locus at which a desired genotype is possible would be even higher at 0.8 . However, the probability that they would have a child with the desired genotype at all 15 loci at which the desired genotype is possible (call this the 'optimal genotype') is vanishingly small at 0.001034 [see Appendix II]. There is 
only a one in 967 chance of having a child with the optimal genotype through natural reproduction.

Our randomly-selected couple could, however, increase their chances of having the child with their preferred genotype by engaging in IVF and preimplantation genetic diagnosis. Suppose that ten embryos are created through IVF, and that the genotypes of each of these embryos at the 15 loci in question can be determined. The probability that one of these embryos will have the preferred genotype will be:

$$
1-(1-0.001034)^{10}=0.010296
$$

This is much higher than the probability for a couple using natural methods. But it is still very low - little more than $1 \%$.

Now suppose now that our couple could create large numbers of embryos using IVG, followed by pre-implantation genetic diagnosis. This would greatly improve the chances of finding an embryo with the optimal genotype. Suppose that 1,000 embryos were created. The chance of finding an embryo with the optimal genotype will now be:

$$
1-(1-0.001034)^{1000}=0.644756
$$

That is, around $64 \%$. Increasing the number of embryos created to 10,000 would increase the likelihood even further to well over $99 \%$ :

$$
1-(1-0.001034)^{10000}=0.999968
$$

Thus, the effect of using IVG could be highly significant. If IVG could be used to create 10,000 embryos for IVF, then this could improve the chances of finding the preferred genotype from just over $1 \%$ to more than $99.99 \%$.

In the case that we have just presented, the couple were interested in selecting 20 single gene traits. But using IVG, it may also become feasible for a couple to select for desired polygenic traits. Thus, we could assume that the 20 loci considered in the above model all contribute to a single polygenic trait (perhaps a disposition to increased intelligence, or reduced risk of heart disease) rather than to 20 distinct single gene traits. In that case key 
results would be as follows. Under our assumptions, a typical couple would be capable of having a child with 15 of the 20 genes that contribute to the polygenic trait (in many cases, having 15 of the 20 genes would be enough to have a significant effect on phenotype).

The chance of the couple having such a child would be just over $1 \%$ with traditional IVF plus selection, but would increased to over $99.99 \%$ if 10,000 embryos could be created using IVG.

\section{Objections}

By enabling the creation of large numbers of gametes and embryos, IVG may allow the selection of traits in future children to a degree that has previously been inconceivable. The use of IVG to aid selection of children would invite different objections depending on whether it occurred at the level of embryos, as in the model described above, or at the level of gametes. In what follows we first address objections to the selection of embryos using IVG, then objections to the selection of gametes via IVG. Finally, we discuss a number of general objections to the use of IVG to create the child with the best chance at the best life. In all cases, we consider only objections to pursuing $\mathrm{PB}$ via the use of IVG. We leave aside objections that are equally applicable to the pursuit of $\mathrm{PB}$ using existing technologies.

\section{Selection of embryos}

Selection at the level of the embryo raises the thorny issue of the moral status of the embryo. While this application of IVG could facilitate the selection of more advantaged children, it would also involve the intentional creation and destruction of very large numbers of embryos. Thus, those who oppose all embryo destruction will also oppose this technology. Those that do not may be forced to decide whether the embryo has some or no moral status.

Each cycle of IVF ultimately results in the destruction of a small number of embryos. While initially embryos that are not implanted may be frozen, these embryos will only be stored for a finite duration and are then either destroyed, donated to another couple, or in some jurisdictions, donated to research. Over 
80,000 embryos are in storage in Australia ${ }^{26}$ with a 5 year storage limit in the state of Victoria. ${ }^{27}$ At the Monash in-vitro fertilisation clinic from 1991-2002, 3053 stored embryos were destroyed, while 425 were donated to another couple. ${ }^{28}$ In the United States, at least 396,526 embryos were in storage as of April 11, 2002, with over 2\% (more than 7,930 embryos) awaiting destruction. ${ }^{29}$ Those who attribute some but not full moral status to the embryo may concede that the destruction of a relatively small number of embryos due to IVF is justifiable to create a child, while opposing the destruction of embryos on a larger scale as would be necessary to pursue PB via IVG.

However there are difficulties with the view that early embryos possess any significant moral status. Millions of embryos are die naturally every year due to spontaneous abortion. Approximately $50 \%$ of embryos spontaneously abort within the first 8-10 days post conception prior to implantation in the uterine wall. ${ }^{30} 56 \%$ of conceptions have resulted in spontaneous abortion by 6 weeks, and $63 \%$ by term. ${ }^{31}$ Based on these estimates 220 million conceptions spontaneously abort every year. ${ }^{32}$ If the early embryo has the same moral status as an adult human, then surely preventing these millions of deaths should be given the highest priority: it would warrant massive international collaboration and resource allocation. However, even knowing the extent of spontaneous abortion, most of us do not think that it is a major world problem on a par with war, famine or disease. Indeed, we think it would be

26 Jishan $\mathrm{H}$ Dean and Elizabeth A Sulivan, 'Assisted Conception Australia and New Zealand 2000 and 2001'. In: AIHW Cat. No. PER 22, Sydney: Australian Institute of Health and Welfare National Perinatal Statistics Unit (Assisted Conception Series No. 7), 2003.

27 Gabor T Kovacs, Sue A Breheny and Melinda J Dear, 'Embryo donation at an Australian university in-vitro fertilisation clinic: issues and outcomes', Medical journal of Australia 178 (3), 2003, 127-9.

28 Ibid.

29 David I Hoffman et al., 'Cryopreserved embryos in the United States and their availability for research', Fertility and Sterility 79 (5), 2003, 1063-9.

30 Henri Leridon, Human Fertility: The Basic Components, Chicago: University of Chicago Press 1977.

${ }^{31}$ Ibid.; Toby Ord, 'The Scourge: Moral Implications of Natural Embryo Loss', The American Journal of Bioethics 8 (7), 2008, 12-9.

32 Ord, 'Moral Implications of Natural Embryo Loss'. 
absurd to give the elimination of spontaneous abortion the priority that is currently given, say, to the elimination of AIDS or cancer. This suggests that we do not really believe that embryos have full moral status. ${ }^{33}$ Indeed, the fact that most of us feel little inclination at all to regard spontaneous abortion as an important public health problem suggests that we assign early embryos no significant moral status. ${ }^{34}$

Some may be concerned that the destruction of embryos on a huge scale would be the beginning of a slippery slope to something more sinister. If we become dismissive about embryo destruction then maybe we will also become blasé about, for example, late abortion. However this concern seems unfounded as research protocols draw a very clear line in embryonic development about how far we can allow embryos to develop and at what point they should be destroyed. While it may be true that it is difficult to identify a point at which moral status begins, this does not mean that no line can be drawn, and effectively enforced by regulators.

We do not claim to have shown that early embryos lack any moral status. We merely wish to emphasise two points. First, that the possible use of IVG in the name of procreative beneficence may force us to decide whether early embryos have any significant moral status. And second, that the view that they do have significant moral status faces some serious, though not necessarily intractable, problems.

\section{Selection of gametes}

Selecting gametes rather than embryos would avoid the destruction of large numbers of embryos, and hence would be significantly less ethically controversial than embryo selection. Gamete selection using IVG may require the creation and subsequent destruction of one embryo per parent, to serve as a source of ES cells, and eventually gametes. Alternatively, it may be possible to eliminate the need for any embryo destruction by deriving induced pluripotent stem (iPS) cells directly from somatic cells. Gametes derived from stem cells, or

33 Ibid.

34 Thomas Douglas and Julian Savulescu, 'Using Unwanted Embryos in Research', EMBO Reports 10 (4), 2009, 307-12. 
naturally-produced sperm from a fertile male, could then be screened to detect their genetic profile, and then the best combination of egg and sperm used to create an embryo.

It would be universally agreed that gametes have little moral status. But do they have any significant moral status? The majority of eggs ovulated during a woman's lifetime go unfertilised, and a much greater number of sperm ejaculated by a male never go on to fertilise an egg. Although gametes have potential to create life, they are frequently allowed to die without fertilisation and this is of no moral concern. Indeed contraception is aimed at preventing gametes from creating life, and this is not normally thought to be controversial. It appears that most people regard the death of gametes prior to fertilisation as being of little moral consequence.

\section{General objections to the pursuit of procreative beneficence via in vitro gametogenesis}

\section{Polygenic traits: which should we maximise?}

As the number of traits being selected increases, the number of embryos or gametes required to allow effective selection increases dramatically. Limits on the number of embryos or gametes that can be produced or tested may thus constrain the number of traits that can be selected. If so, it will be necessary to prioritise traits. This may be difficult. Is it more important to prevent the development of heart disease or Crohn's disease? Obesity or diabetes? To select for a disposition to intelligence or to cheerfulness? There are no easy answers to these questions. However technological advances may alleviate the difficulty, if they allow the production and screening of massive numbers of gametes or embryos.

\section{Determining the good life: which traits to select for?}

The good life is highly complex. This creates difficulties even for the selection of single traits. Suppose we are considering whether to select one trait $T$, where all other traits will be determined through natural means. Whether it is good for the child to possess $T$ may depend on what other traits she ends up having. Thus, for example, it seems plausible that whether it is good to 
possess a disposition to perfectionism depends on whether one also possesses a disposition to impatience: an impatient perfectionist is likely to be very dissatisfied, whereas a patient one may achieve great things (we are not suggesting that it is, or will soon become, possible to select for these traits). For this reason, one might think that it would be unethical for a parent to select for perfectionism.

But now suppose that it is possible to select for multiple traits through IVG. In some ways, this alleviates the problem. A parent who selects for perfectionism can also select for patience (though of course environmental factors might well intervene to prevent one of the dispositions from being manifest). However, a new problem now arises. Now, rather than merely deciding whether it is good to have one trait or not, we have to rank various combinations of traits: is it better to be perfectionist and patient, or impatient and easily satisfied? If we are only selecting two traits, this task may be manageable. But suppose we are instead selecting twenty traits. It is likely to be extremely difficult to rank the four hundred possible combinations of traits, and any ranking we do reach will be highly uncertain.

However, it does not follow from the fact that it is difficult to determine what combinations of traits are conducive to a good life that we should give up on procreative beneficence. In fact parents already face a similar problem in deciding how to raise and educate their children. Should they try to inculcate a competitive and determined disposition, or a cheerful and relaxed one? Nevertheless, we do not think that parents should abstain from exerting any influence on the character and abilities of their children. Presumably this is because there are some traits - such as imagination, intelligence, empathy, the ability to delay gratification, and the ability to identify grossly immoral conduct - which, at least when possessed to a moderate degree, are almost always conducive to the good life under environmental conditions that can reasonably be expected to prevail.

\section{The quest for perfection}

How far should we go in pursuit of the best possible child? While interference in reproduction and embryo destruction with the goal of preventing children 
being born with debilitating diseases may seem justified, many would argue that the use of IVG to select for multiple non-disease traits is not.

It could be argued that the more traits we select for, the more parental expectations there will be for the child to live up to. However parents already have hopes, dreams, aspirations and expectations of their children regardless of their genetic makeup. It is not clear that selection of children with multiple desirable traits using IVG will raise these expectations. Furthermore, it is pertinent to remember that parents have a propensity to love their children unconditionally, irrespective of their physical or intellectual characteristics and achievements. In any case, the aim of PB is to give the child the best life, rather than to satisfy parental desires. If parents select traits which do not increase the child's expectation of a good life - whether because the very act of selection is likely to create unreasonable expectations in the parents, or because the parents are simply mistaken about what will be good for the child - then they are not following PB at all. Good parents will always strive for what is best for their children, be it through providing them with the best education, experiences, living situation or other means. Applying the principle of PB and the selection of multiple traits is merely another means of providing children with as many tools possible to construct a good life.

The more traits that are selected for in the pursuit of perfection, the more control we gain over what our children will be like and the kind of life that they might lead. By doing this it has been argued that we remove the element of the unknown and the unexpected, the 'openness to the unbidden', elements that are a key part of existence, parenthood and a good life. ${ }^{35}$ However our genetic makeup determines only a small part of our existence. Our upbringing, environment and experiences all play a vital role in shaping the people that we become. Furthermore the human genome is made up of such vast numbers of genes that the fraction being selected for would be a tiny percentage. Our genetic makeup can certainly give us advantages in life, but does not shape our entire existence. Instead it provides us with the initial framework on which

35 Michael J Sandel, 'The case against perfection: what's wrong with designer children, bionic athletes, and genetic engineering', The Atlantic Monthly 292 (3), 2004, 51-62, 56. 
to build our lives and character, the selection or manipulation of which may broaden rather the limit the opportunities available to us and hence increase the number of different paths which our lives could take.

\section{Eugenics}

Some may be concerned that the use of IVG to pursue the principle of PB would approach population eugenics in a way that previous potential methods of child selection did not. The more traits we can select for, the greater the impact on the lives of our children, but also the greater the impact on the population gene pool. Not only could the most desirable traits be selected for, but the use of this technology could mean that certain genetic conditions would be eradicated from the gene pool altogether. This could be likened to the introduction of vaccination, which has meant that many diseases, such as polio, have virtually ceased to exist in the population. Most would herald the discovery of vaccination by Edward Jenner as one of the great medical breakthroughs, with vaccination now an accepted part of healthcare. Although there are some that oppose vaccination, the majority do not question its benefits. Selection technology and genetic manipulation of the germline could bring disease prevention to a whole new level. We would be able to prevent disease by erasing genetic mutations in future generations, with the benefit reaped by all and without significant sacrifice by the individual. Like vaccination this would benefit both the individual and the group simultaneously. Surely from a purely medical perspective this would be a welcomed and celebrated breakthrough. Indeed modern medicine aims to create a disease-free population. But is striving to eradicate genetic diseases from the gene pool eugenics? And if so, does it follow that it is unethical?

It is important to emphasise the differences between the pursuit of procreative beneficence through IVG and clearly objectionable eugenics programmes conducted in the past, for example, in Nazi Germany. The definition of eugenics is the improvement of the gene pool, which in itself is not necessarily an evil. The Nazis, however, strove to bring this about through killing, restrictions on reproduction and coercion. Nazi practices were also based on misguided concepts of disease and disability, were driven by racist and imperialist motives. 
The wrongness of Nazi eugenics seems to be explained best by the immorality of the means that were used, and the beliefs and intentions which motivated their use. It is not clear that parents striving to pursue procreative beneficence could be criticised on the same grounds. Consider the case of an individual couple who decide to select among in vitro derived gametes in order to avoid having a disabled child. Whereas the Nazis wished to improve their gene pool to give Aryans a survival advantage over other nations or races, the desire of this couple presumably stems from no such motives, but merely the desire to give their child the best life possible. Similarly, whereas Nazi eugenics involved murderous and coercive means, in the case of the couple, no one is harmed or coerced. As Jonathan Glover has noted, when parents elect to prevent disability, '[i]nstead of the subordination of the individual to the social Darwinist struggle for survival, there is compassion for the future child. Instead of coercion by the state, there is respect for parental choice. And ... these parental choices are compatible with an attitude of equality of respect for everyone'. ${ }^{36}$

\section{Limitations}

In the instance of embryo or gamete selection, the traits that can be selected for are limited to the genetic pool of the couple. This means that super-humans are unlikely to be created via this method; at most a child will be created with the best possible combination of genes offered by their parents. This could be seen as a limitation of this technology, or equally as a positive. Any child created via these methods would be a genuine product of their parents. In light of this, it is interesting to consider whether the pursuit of the best child might become of such great importance in the future as to lead to genetic matchmaking - the selection of a partner based on their genetic compatibility for reproduction.

Many will dismiss this ethical debate on the grounds that the technology is not yet available and may never be possible. At this point no laboratory has successfully created functional human eggs or sperm. Furthermore, the function of the genetic sequencing of many genes has not yet been discovered and this must be known before any screening for selection purposes or genetic

36 Jonathan Glover, Choosing Children: Genes, Disability, and Design, New York: Oxford University Press: 2006, 28. 
manipulation could take place. It is true that thus far the science has not caught up to this debate. But we must remember that it is imperative for ethical debate to precede the science so that an ethical framework is in place once the technology arrives. Science often progresses in leaps and bounds, and has taken society by surprise in the past and will do so again. Furthermore, the advent of IVG is far from unforeseeable.

\section{Conclusion}

We have argued that the advent of IVG may greatly aid parents in selecting the children with the best chance at having the best life. In our model, a typical couple wishing to select for twenty single gene traits will be capable of having a child with 15 of those traits. However, even if the couple employs IVF and then selects among ten embryos, the chance of finding an embryo with all 15 traits will be less than $1 \%$. On the other hand, if IVG were used to produce 10,000 embryos, the probability of finding an embryo carrying all 15 traits would rise to over $99 \%$. If large scale pre-fertilisation genetic testing of gametes were possible, similar results could be achieved by producing large numbers of gametes using IVG.

The use of IVG as an aid to procreative beneficence raises several ethical issues. IVG combined with selection at the level of the embryo would almost certainly result in the destruction of huge numbers of embryos. This prospect may force us to decide whether the early embryo possesses any significant moral status. The need for embryo destruction could be avoided if it were possible to select at the level of gametes rather than embryos. Further objections to the pursuit of PB via IVG maintain that it will be difficult to prioritise traits for selection, that interaction between traits will make it difficult to determine which traits would give a child the best life prospects, that such uses of IVG would be excessively perfectionist, that they would constitute an objectionable form of eugenics, and that it IVG technologically unrealistic. We have argued that these objections are unpersuasive. 


\section{Appendix I. Number of loci at which a randomly selected couple would be capable of having a child with the desired genotype}

Suppose a couple would like to have a child that is $r r$ at some recessive locus. What is the probability that they are capable of doing so? If we make the simplifying assumption that the genotypes $R R, R r, r R$, and $r r$ at this locus are equally prevalent in both the male and female population, then the chance that any individual is capable of passing on an $r$ allele to his or her child will be 0.75 ( $25 \%$ of the population will be $R R$ ). For a couple to have an $r r$ child, each member of the couple will have to be capable of passing on an $r$ allele, and the probability of this, in a random couple, will be $0.75 \times 0.75=0.5625$. The couple will have a $56 \%$ chance of being capable of having an $r r$ child.

\begin{tabular}{|c|c|c|c|c|}
\hline $\begin{array}{c}\text { Parent } \\
\text { genotypes }\end{array}$ & $\mathbf{R r}$ & rR & $\mathbf{R r}$ & $\mathbf{R} \mathbf{R}$ \\
\hline $\mathrm{Rr}$ & & \multirow{3}{*}{\multicolumn{2}{|c|}{ rr child possible }} & \\
\hline rR & & & & \\
\hline $\mathbf{R r}$ & & & & \\
\hline RR & & \multicolumn{2}{|c|}{ rr child not possible } & \\
\hline
\end{tabular}

Now suppose that there are ten different recessive loci at which the randomly selected couple would like to have an $r r$ child. Since the probability that the couple can have an $r r$ child at any one of these loci is $56 \%$, we would expect that the couple would be capable of having an $r r$ child at 6 of the 10 loci.

Now consider a dominant locus at which a couple would like to have a child who is $D D, D d$, or $d D$. All couples will be capable of having a child with one of these genotypes except couples who are both $d d$. 


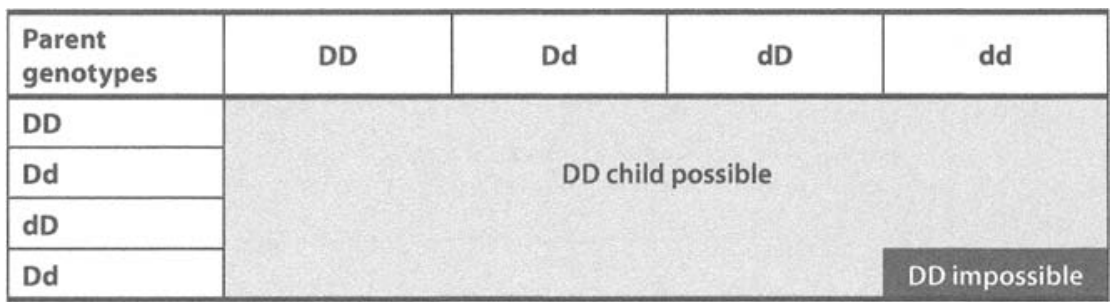

Again, assuming that the four different allele combinations are equally prevalent at the locus, then a couple's chance of both being $d d$ will be $0.25 \mathrm{x}$ $0.25=0.0625$. The probability that the couple will be capable of having a child with the desired genotype will thus be $1-0.0625=0.9375$. We would expect a randomly selected couple to be capable of having a child with the desired genotype at 9 of the 10 loci.

\section{Appendix II. Probability with which a couple would have a child with the desired genotype at all 15 loci where the desired genotype is possible}

Consider one of the recessive loci at which a couple is capable of having a child with the desired $r r$ genotype. For this to be possible, each parent must be either $r r, r R$, or $R r$. Assuming the couple has been randomly selected from a population in which all allele combinations are equally common, then each of these three combinations will be equally likely for each parent. The chance of a child inheriting an ' $r$ ' allele from one parent will thus be $2 / 3$, and the same for the other parent. There is thus a $2 / 3 \times 2 / 3=4 / 9=0.4444$ chance that the child will inherit an $r$ allele from bother parents, and thus be $r$.

\begin{tabular}{|c|c|c|c|c|}
\hline $\begin{array}{l}\text { Parent } \\
\text { genotypes }\end{array}$ & rr & $r R$ & $\mathbf{R r}$ & $\mathbf{R R}$ \\
\hline rr & \multirow{3}{*}{\multicolumn{3}{|c|}{$4 / 9$ chance that the child will be rr }} & \\
\hline$r R$ & & & & \\
\hline $\mathbf{R r}$ & & & & \\
\hline RR & \multicolumn{3}{|c|}{ rr child not possible } & \\
\hline
\end{tabular}


The chance that the couple will be capable of having a child that is $r r$ all six of the recessive loci at which the couple is capable of having an $r r$ child will be:

$$
0.444444^{6}=0.007707
$$

We can do a similar calculation for the 9 dominant loci at which the couple is capable of having a child with the desired genotype. A couple will be capable of having a child with one of the desired $D D, D d$, or $d D$ genotypes unless both parents are $d d$. Thus, if the couple is capable of having a child with the desired genotype at a dominant locus, it must be the case either that:

(a) Neither parent is $d d$ at that locus, or,

(b) One parent is $d d$ at the locus, but the other is not

Assuming that our couple has been randomly selected from a population in which all four allele combinations are equally common, but that the couple is indeed capable of having a child with one the desired genotypes (that is, the parents are not both $d d$ ) then there will be a $9 / 15$ chance that (a) is true, and a $6 / 15$ chance that (b) is true. If (a) is true, then the child has a $1 / 3 \times 1 / 3=1 / 9$ chance of inheriting a $d$ allele from both parents, so she has a $1-1 / 9=8 / 9$ chance of inheriting a $D$ allele from at least one parent. If (b) is true then the child has a $2 / 3$ chance of inheriting at $D$ allele from one parent, and a zero chance of inheriting a $D$ from the other. So the chance of the child inheriting at least one $D$ allele is $2 / 3$.

\begin{tabular}{|l|c|c|c|c|}
\hline $\begin{array}{l}\text { Parent } \\
\text { genotypes }\end{array}$ & DD & Dd & dD & dd \\
\cline { 1 - 1 } DD & Possibility (a): $8 / 9$ chance that child is not dd & $\begin{array}{c}\text { Possibility (b): } \\
2 / 3 \text { chance that } \\
\text { child is not dd }\end{array}$ \\
\cline { 1 - 1 } Dd & Possibility (b): $2 / 3$ chance that child is not dd & DD impossible \\
\hline dD
\end{tabular}

The overall probability that the couple will have a child with one of the desired genotypes (i.e. at least one $D$ allele) will be:

$$
9 / 15 \times 8 / 9+6 / 15 \times 2 / 3=23 / 30=0.8
$$


So the chance that the couple will have a child with the desire genotype at a single given locus at which they are capable of having such a child will be 0.8 . The chance that they will have a child with the desired genotype at all 9 loci at which they are capable of having such a child will be:

$$
0.8^{9}=0.134218
$$

We can now calculate the probability that the couple will have a child that has the desired genotype at all 15 loci (6 recessive and 9 dominant). It is:

$$
0.007707 \times 0.134218=0.001034
$$

\section{Acknowledgement}

I thank the Wellcome Trust (grant number WT087211) for their funding. 